\title{
NUMERICAL STUDY OF DROPLET FORMATION IN A Y-JUNCTION MICROCHANNEL
}

\author{
Mouhamadou M.L. Diouf \\ ENSIAME - École Nationale Supérieure d'Ingénieurs en Informatique Automatique Mécanique Énergétique \\ Électronique Engineering School in Valenciennes, France; e-mail: alamine95@yahoo.fr
}

OLDRICH J. ROMERO

Petroleum Engineering Undergraduate Program and Graduate Energy Program, Federal University of Espirito

Santo - UFES, ES, Brazil; e-mail: oldrich.romero@ufes.br

\begin{abstract}
This study investigates the formation process of droplets in a Y-junction microchannel using two immiscible fluids: water as the continuous fluid and oil as the dispersed phase. We have examined the influence of the capillary number, flow rate ratio and viscosity ratio between the two fluids; parameters which determine the length and generation frequency of the droplets. Numerical simulations have been performed using the software Ansys Fluent with the interface capture method Volume of Fluid (VOF) for solving the governing equations. Three different algorithms have been tested for the pressure-velocity coupling: SIMPLE, SIMPLEC and PISO. The results are quite similar for SIMPLE and SIMPLEC, however it turned out that PISO is a better algorithm to solve the two-phase flow. Additionally, another Y-junction is coupled in the initial geometry to observe a symmetric breakup of the droplets and their formation is explained using the pressure field and the velocity field.
\end{abstract}

Keywords: microfluidic, Y-junction, two-phase flow, numerical simulation, VOF

\section{Introduction}

Microfluidics can be defined as science which studies the behaviour of fluids in micro-channels (dimensions varying from $10 \mu \mathrm{m}$ to $500 \mu \mathrm{m}$ ). Micro-channels becomes increasingly used every day due to a microminiaturization of biomedical devices (Micro-electromechanical system MEMS technologies) (Lih and Miao, 2015). Microfluidics have many applications such as: DNA fabrication, encapsulation of cells, drug delivery and protein crystallization (Dolomite, 2015). For these applications, it is important to control as well as possible the geometry of the generated droplets. There are many ways of making droplets depending on the geometry used, as it was shown and briefly explained by Dolomite (2015).

In this study, we will focus on a segmented flow device. For that, a Y-junction (Fu et al., 2011, 2014; Cong et al., 2014) is used in order to generate periodic droplets. One continuous fluid is injected into the first channel and a dispersed fluid is injected into the secondary channel, both fluids are incompressible and immiscible.

Several parameters such as the viscosity ratio between the two fluids, the flow rate ratio, the capillary number, the aspect ratio of the geometry, the sizing of the meshing between others, can affect considerably droplet generation. This study investigates the influence of those parameters in the formation process by checking for every case the variation in length of generated droplets and the pressure field. To have a better understanding of the phenomena, a comparison between the predicted lengths of droplets by scaling proposed by Garstecki et al. (2006) and the numerical ones will be made. Tarchichi et al. (2013) showed the influence of the capillary number (Ca). Depending on its value, 3 regimes of droplets can occur: the squeezing regime for low value of Ca, 
the dripping regime as $\mathrm{Ca}$ increase and the jetting regime at higher value of Ca. The effects of the viscosity ratio on the formation of the droplet are discussed in Tice et al. (2004). According to that reference, droplets are formed when the viscosity of the dispersed fluid is significantly higher than that of the continuous fluid.

All numerical simulations have been performed using the software Ansys Fluent 15.0 with the volume-of-fluid (VOF) method. In every case, the continuous phase which is injected in the main channel is water, and the dispersed phase is oil.

\section{Problem description}

In this study, we are using a Y-geometry (Fig. 1a) which is similar to that of Liu et al. (2015). This geometry have two inlets with the same width $h$. Both extend until they meet in a junction forming channels with two parallel plates whose distances are the same, $4 h$. The length of the main channel is taken to be $36 h$ for the development of periodic emulsions of oil in water. This geometry is then modified by adding two orthogonal channels at the end of the main channel (Fig. 1b) (Fu et al., 2011, 2014; Cong et al., 2014); channels which have the same width $h / 2$ and the same length $10 h$. In all the work that follows, $h=0.1 \mathrm{~mm}$. Water with a viscosity $\mu_{w}$ of $1 \mathrm{cP}$ $(0.001 \mathrm{~Pa} \cdot \mathrm{s})$ is injected at inlet 2 and oil with a viscosity $\mu_{o}$ of $10 \mathrm{cP}(0.01 \mathrm{~Pa} \cdot \mathrm{s})$ in inlet 1 ; both are moving along their channel until they meet in the junction, named here Y-junction, and moving forward along the main channel. The density of water $\rho_{w}$ and of oil $\rho_{o}$ are respectively $1000 \mathrm{~kg} / \mathrm{m}^{3}$ and $900 \mathrm{~kg} / \mathrm{m}^{3}$. The interfacial tension between water and oil $\left(\sigma_{w / o}\right)$, which is an important parameter for the formation of droplets, is equal to $0.0003 \mathrm{~N} / \mathrm{m}(30 \mathrm{dyn} / \mathrm{cm})$

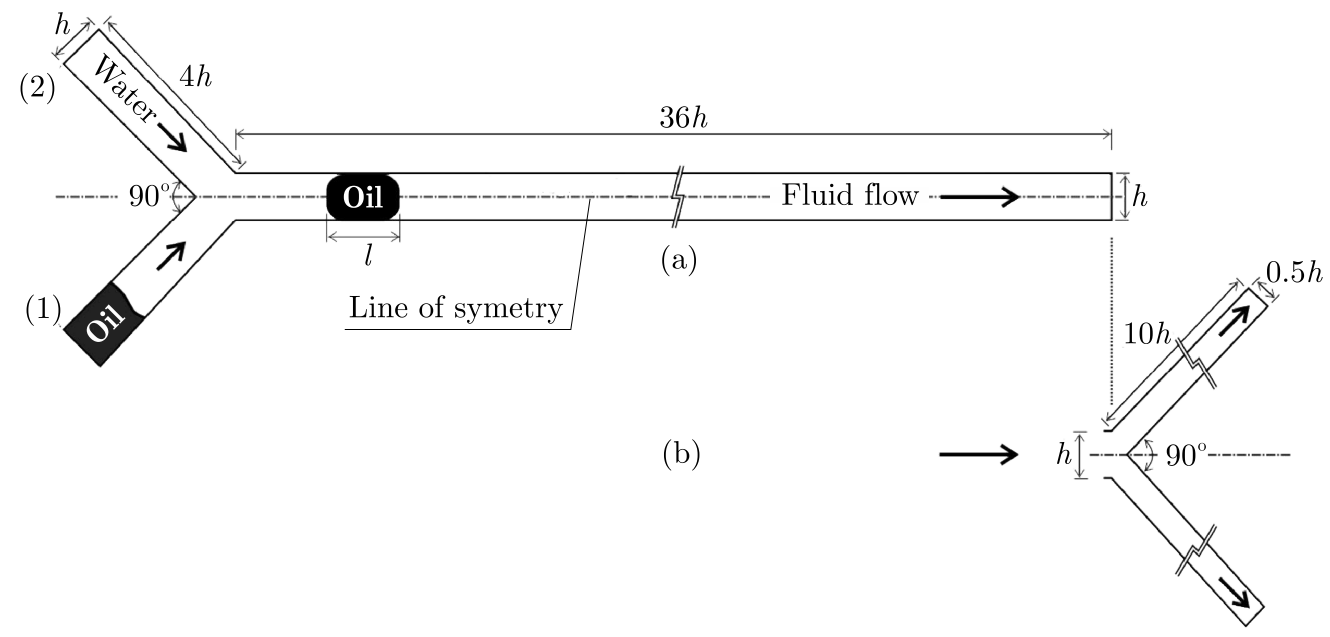

Fig. 1. Schematic view, not in scale, of the Y-junction geometry used in this work

\section{Mathematical approach of the liquid-liquid flow problem}

The volume fraction of water $\alpha_{w}$ and oil $\alpha_{o}$, both ranging between 0 and 1 , determines the interaction between the two fluids in the oil mobilization process, and is obtained using the multiphase model Volume of Fluid - VOF developed by Hirt and Nichols (1981). This model solves mass conservation and momentum equations considering the volume fraction present in every cell from the physical domain of the problem and for different instants. The proposal consists in calculating the volume fraction of the less dense phase, oil in this case 


$$
\frac{\partial}{\partial t}\left(\alpha_{o} \rho_{o}\right)+\nabla \cdot\left(\alpha_{o} \rho_{o} \mathbf{v}\right)=0
$$

Considering the presence of two phases, water $w$ and oil $o$, the sum of the volume fractions at each point of the domain must be unitary, i.e.

$$
\alpha_{w}+\alpha_{o}=1
$$

The water volume fraction is obtained directly from Eq. (3.2) as $\alpha_{w}=1-\alpha_{o}$, since the oil volume fraction is already known from Eq. (3.1).

Density $\rho_{w o}$ and viscosity $\mu_{w o}$ in Equation (3.5), are mixture properties which vary within the flow domain and are computed by the volume fraction weighted average as

$$
\rho_{w o}=\alpha_{w} \rho_{w}+\alpha_{o} \rho_{o} \quad \mu_{w o}=\alpha_{w} \mu_{w}+\alpha_{o} \mu_{o}
$$

The continuity equation, which describes mass conservation in the whole domain, i.e., in the water and oil region, where the velocity vector is $\mathbf{v}$, is given by

$$
\nabla \cdot \mathbf{v}=0
$$

Similarly, the linear momentum conservation principle is applied in the whole domain

$$
\frac{\partial}{\partial t}\left(\rho_{w o} \mathbf{v}\right)+\nabla \cdot\left(\rho_{w o} \mathbf{v v}\right)=-\nabla p+\nabla \cdot\left(2 \mu_{w o} \mathbf{D}\right)+\rho_{w o} \mathbf{g}+\mathbf{f}_{i n t}
$$

where $\rho_{w o}$ is the average density, $\mathbf{g}$ represents gravity acceleration and $\mathbf{D}=0.5\left[\nabla \mathbf{v}+(\nabla \mathbf{v})^{\mathrm{T}}\right]$ is the strain rate tensor. The source term $\mathbf{f}_{\text {int }}$ in momentum equation, added for modeling water/oil interfacial tension $\sigma_{w o}$ effects on fluid motion, is described by the Continuum Surface Force (CSF) model proposed by Brackbil et al. (1992) and expressed by

$$
\mathbf{f}_{i n t}=\sigma_{w o} \frac{\rho_{w o} \kappa \nabla \alpha_{o}}{\frac{1}{2}\left(\rho_{w}+\rho_{o}\right)}
$$

this term is known in the literature as the Continuum Model Surface Force - CSF, where $\kappa$ is the curvature of the interface, $\rho_{w}$ is density of water and $\rho_{o}$ is density of oil.

The boundary conditions which define the solution domain, are applied as shown in Fig. 2, where:

(1) In inlet 1 , the average velocity of oil $V_{o}$ is taken to be equal to $0.00009 \mathrm{~m} / \mathrm{s}$;

(2) In inlet 2, water flows with a velocity $V_{w}$ of $0.000417 \mathrm{~m} / \mathrm{s}$;

(3) In the outlet, the gauge pressure is equal to $0 \mathrm{~Pa}$;

(4) The interface is applying a force, defined previously by Eq. (3.6); and

(5) Finally, no-slip and zero diffusive mass flux are specified in the walls.

Also, as an initial condition used in this transient problem, the microchannel is filled with the primary phase, which is in this case water represented by white colour.

In order to deal with the interaction between the two immiscible fluids described in the previous section, some parameter that identifies the shear forces acting on the interface needs to be defined. According to Coelho et al. (2016) and Santos et al. (2016), the interfacial tension is an important property that contributes to the form adopted by the droplets through the interface. In many cases, the convergence of the solution depends on its value. In addition, several other parameters such as viscosities and velocities of both fluids, channels width, numerical parameters, can influence the droplets formation and convergence of the solution process. Their influence 
$(2)$

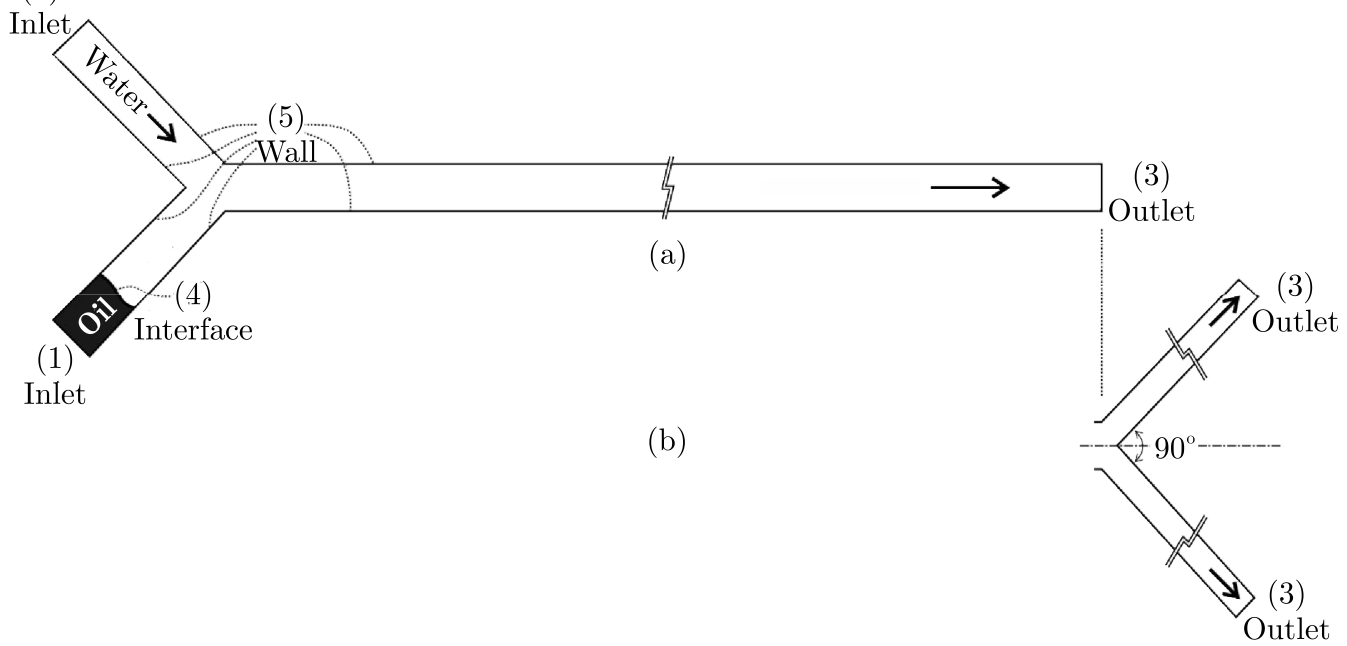

Fig. 2. Boundary conditions at the Y-junction geometry used in this work

in the fluid flow can be better understood using dimensionless numbers, as represented by Eqs. (3.7) to (3.15), the following:

(i) The capillary number Ca reflects the ratio between the viscous forces and the capillary forces. The first term depends on the viscosity of the dispersed fluid, in this case oil $\mu_{o}$ which is injected with an average velocity $V_{o}$, and the second term is the interfacial tension between the two fluids $\sigma_{w o}$

$$
\mathrm{Ca}=\frac{\mu_{o} V_{o}}{\sigma_{w o}}
$$

(ii) The comparison between the more viscous fluid, oil $\mu_{o}$, and less viscous fluid, water $\mu_{w}$, is represented by the dimensionless viscosity ratio $\mu_{R}$ defined by

$$
\mu_{R}=\frac{\mu_{o}}{\mu_{w}}
$$

(iii) The Reynolds number Re represents the relationship between the inertia forces (represented by the group $\rho_{o} V_{o} h$ ), with the viscous forces of the dispersed fluid, which is oil. The expression is

$$
\operatorname{Re}=\frac{\rho_{o} V_{o} h}{\mu_{o}}
$$

where $h$ is the characteristic length chosen to represent the flow, which is in this case the width of the channel through which oil flows. In all simulations performed in this study, the Reynolds number is relatively small $(\operatorname{Re} \leqslant 1)$ meaning that the inertial forces are almost insignificant when compared with the viscous forces.

(iv) The way in which droplets are formed throughout the main channel is strongly influenced by the contact angle $\theta_{c}$ of the fluids that are in contact with the walls. Thus, the wettability (or contact angle) indicates which phase is preferably wet to the walls. According to Jamaloei et al. (2011), a contact angle equal to zero $\theta_{c}=0$ indicates complete wetting of the denser phase (water), $\theta_{c}=\pi$ rad indicates complete wetting of the less dense phase (oil) and an angle of $\theta_{c}=\pi / 2 \mathrm{rad}$ indicates that neither of the phases wet preferably than the other. In this study, the contact angle is $\theta_{c}=0 \mathrm{rad}$.

(v) The flow rates of oil and water are respectively noted $q_{o}$ and $q_{w}$, and the ratio between them is described by

$$
q_{R}=\frac{q_{o}}{q_{w}}
$$


(vi) According to Garstecki et al. (2006), the length $l$ of the droplets (Fig. 1a) depends on the flow rate ratio between the two fluids $q_{R}$, the width $h$ of the main channel and the constant $\alpha$ depending on the geometry used

$$
\frac{l}{h}=1+\alpha q_{R}
$$

(vii) Based on Coelho et al. (2016) and Santos et al. (2016), one of the main parameters described in the literature to obtain the convergence of the solution with the explicit approach to time-dependent problems is the Courant number, defined by

$$
C_{O}=\frac{V_{m} \Delta t}{\Delta x}
$$

where $V_{m}$ is the average velocity of the dispersed fluid $\left(V_{m}=V_{o}\right), \Delta x$ is the size of a representative element of the mesh and $\Delta t$ is the time-step size. Equation (3.13) which is derived from Eq. (3.12), is known as Courant-Friedrichs-Lewy (CFL)

$$
\Delta t_{C F L}=\frac{\operatorname{Co} \Delta x}{V_{m}}
$$

the Courant number should be less than $1(\mathrm{Co}<1)$ to assure the convergence of iterative solution (Bethke, 2008).

In order to highlight the effects of interfacial tension and viscosities, other equations to determine the time step are presented. One of them is the relation proposed by Brackbill et al. (1992) which establishes the time step as a function depending on the mass density of oil and the interfacial tension

$$
\Delta t_{B}=\sqrt{\frac{\rho_{o} \Delta x^{3}}{2 \pi \sigma_{w o}}}
$$

another example is the equation proposed by Galusinski and Vigneaux (2008), where the time step $\Delta t_{V}$ is depending on the viscosity of oil and the interfacial tension

$$
\Delta t_{V}=\frac{\mu_{o} \Delta x}{\sigma_{w o}}
$$

\section{Numerical solution of the governing equations}

To solve numerically the set of governing equations, Eqs. (3.1), (3.4) and (3.5), it is necessary to discretize it in order to obtain a system of algebraic equations (Patankar, 1980).

\subsection{Discretization algorithms}

The discrete version of governing equations is solved using the commercial software Ansys Fluent 15.0 with the VOF technique. In this process, one of the key steps is the treatment of the pressure field. In fact, gradients in the pressure appear in the Navier-Stokes equations. However, since we are using an incompressible fluid, the density of each fluid is constant, thus the pressure can not be calculated directly from the equation of state. An alternative way to determine the pressure field is to couple the pressure and the velocity. Three pressure-velocity coupling schemes are available in Fluent: SIMPLE (Semi-Implicit Method for Pressure-Linked Equations); SIMPLEC (SIMPLE Consistent) is an improved version of SIMPLE, and PISO (Pressure Implicit with Splitting of Operators) is using a similar method as SIMPLE and SIMPLEC with a better correction. All three algorithms are used for the pressure-velocity coupling. For more details see 
Ferziger and Peric (2002) and Versteeg and Malalasekera (1998). Their effects in the flow field behaviour will be presented and discussed in the results Section.

The PRESTO schemes (Pressure Staggering Option) and Second Order Upwind are used respectively for the interpolation of pressure and velocity. For the evaluation of gradients, the Least Squares Cell based method is used. It is quite accurate and computationally less expensive compared to other methods.

The Geometric Reconstruction (Geo-Reconstruct) method, used for oil and water volumetric fractions, allows good definition of the liquid-liquid interface of the Y-junction since it uses a linear approach to represent the interface between the fluids.

\subsection{Mesh test}

For the spatial discretization of the domain, three different meshes have been tested, as is shown in Fig. 3 (left) where the maximum size of the rectangular elements is gradually decreased, which means a refined mesh. The first one (Fig. 3a) is the coarsest mesh, containing 1106 rectangular elements and referred as mesh 1. In Fig. 3b, corresponding to the regular mesh contains 4423 rectangular elements. The size of the elements for this mesh is in the range between $0.001 \mathrm{~mm}$ and $0.01 \mathrm{~mm}$. Reducing again the size of the rectangular elements leads to the most refined mesh among them (Fig. 3c). This mesh contains 8838 elements. The parameters for each mesh is detailed in Table 1.

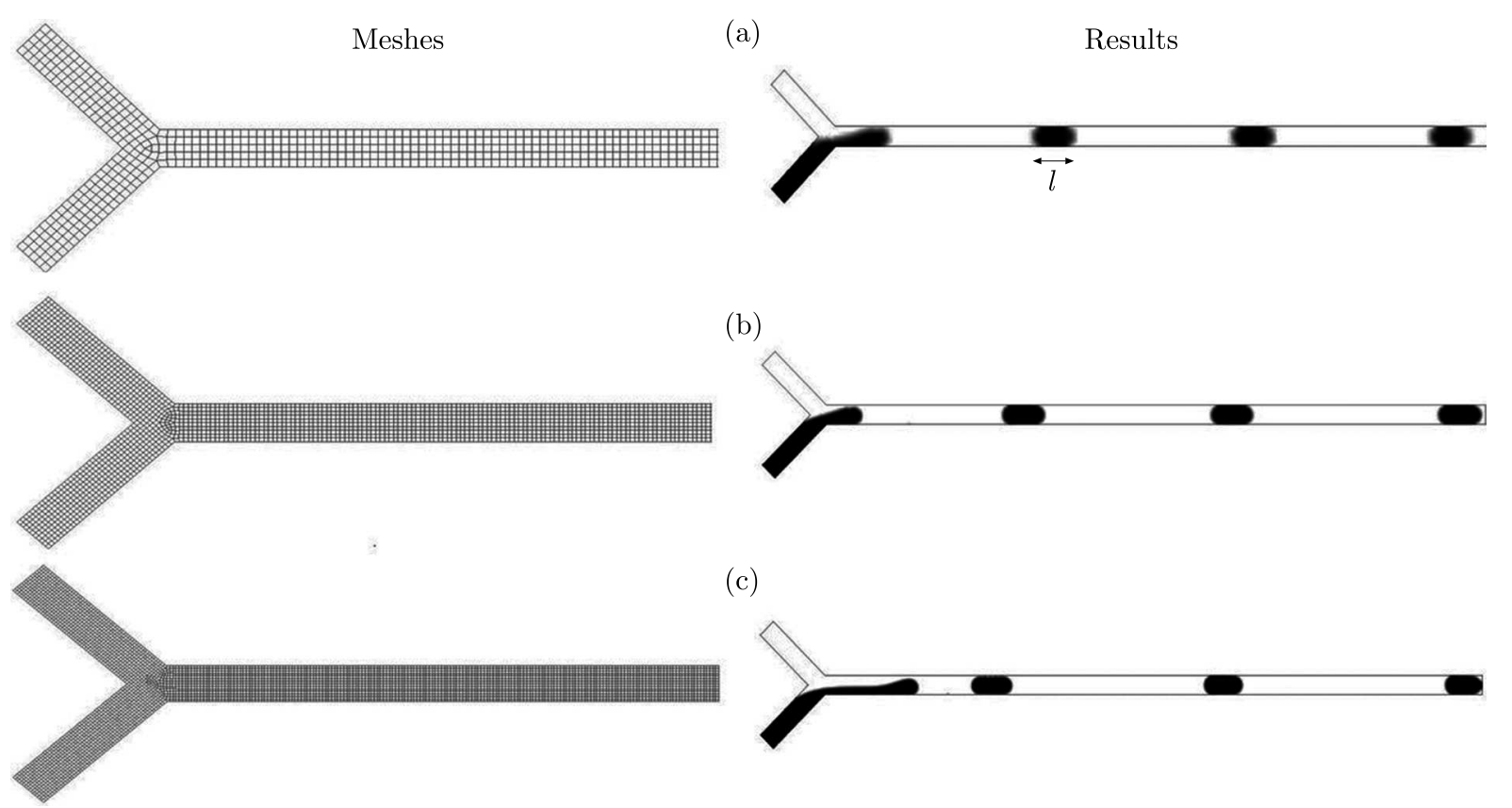

Fig. 3. Mesh (left) and water volume fraction (right) for 3 meshes tested at $13.8 \mathrm{~s}$ : (a) coarse mesh, (b) regular mesh, and (c) refined mesh

Table 1. Parameters of the three meshes

\begin{tabular}{|c|c|c|c|}
\hline Parameters & Mesh 1 & Mesh 2 & Mesh 3 \\
\hline \hline Number of elements & 1106 & 4423 & 8838 \\
\hline Minimum size $[\mathrm{mm}]$ & 0.001 & 0.001 & 0.001 \\
\hline Maximum size $[\mathrm{mm}]$ & 0.02 & 0.01 & 0.007 \\
\hline
\end{tabular}


Simulation has been performed for each mesh, with the same boundary conditions. The results are shown in Fig. 3. In the right side of this figure, it can be seen that droplets with similar regularity and shape are obtained for all three meshes. However, the position where the droplet is formed is different from one mesh to another. The way in which droplets are being formed will be explained in the next Section.

In order to choose a better mesh, a comparison between the predicted length of the droplet and the ones obtained for meshes 1 and 2 has been made. For $q_{R}=0.2158$ and $h=0.1 \mathrm{~mm}$, the length of the droplets obtained from Garstecki equation, Eq. (3.12), with $\alpha=6.5$ is $l_{\text {Teo }}=l=0.24 \mathrm{~mm}$. For the three meshes, the length of the droplet obtained numerically $l_{\text {Num }}$ is shown in Fig. 4. The relative error is defined as $\varepsilon_{R}=100\left|l_{N u m}-l_{\text {Teo }}\right| / l_{\text {Teo }}$. After calculations $\varepsilon_{R_{\text {Mesh } 1}}=8.33 \%, \varepsilon_{R_{\text {Mesh } 2}}=4.17 \%$ and $\varepsilon_{R_{\text {Mesh } 3}}=12.50 \%$. The relative errors are smaller for Mesh 2. From these results, it is clear that the regular mesh is the most suitable for this geometry, compared to the others. It will be used in the next simulations.
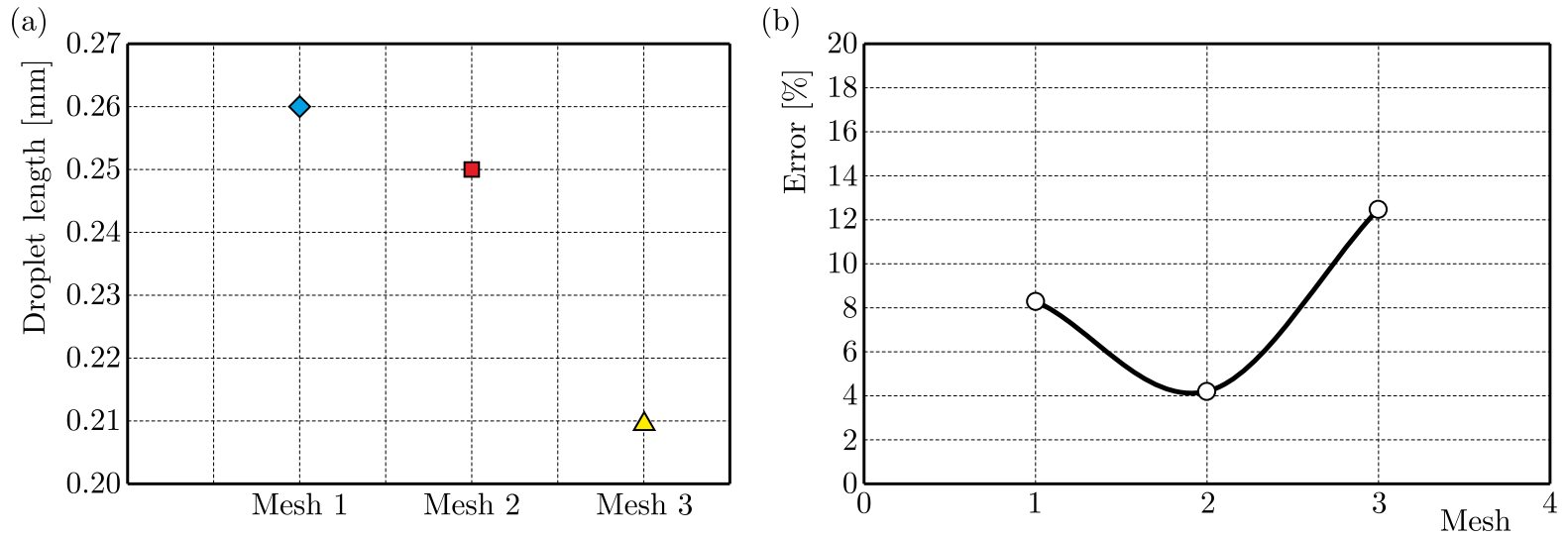

Fig. 4. (a) Length of the generated droplet $l_{N u m}$ for each mesh; and (b) comparison between length of the droplet obtained by numerical approach $l_{N u m}$ with analytical equation $l_{N u m}$

Although mesh comparisons reported in Fig. 4a is apparently not converging to a constant solution, our main criterion to select the mesh number 2 is the best representation of the analytical equation, Eq. (3.11). The scaling relation proposed by Garstecki et al. (2006), obtained by experiments, is commonly applied and used as a reference in the literature. This procedure is adopted because refined mesh were tested, increasing considerable the time computing required to obtain solutions which are not completely converging to a constant droplet length. The comment of Qian and Lawal (2006) "...All these complexities make VOF multiphase flow simulation computationally expensive, and convergence difficult to achieve when compared to its single phase counterpart. In fact, multiphase flow is perhaps the most difficult topic in the CFD simulation...", it is appropriate at this time.

\subsection{Selection of the time-step}

In this transient problem, the simulation time $t_{n}$ is determined from the previous time $t_{n-1}$ by the equation $t_{n}=t_{n-1}+\Delta t$. The advance in time is repeated until $t_{n}=t_{\text {final }}$, which represents the end of the simulation when $n=N$, where $N$ is the number of time-steps. The choice of the time-step $\Delta t$ is extremely important for problems with liquid-liquid interfaces. To assure the convergence of the solution, the Courant number should be less than one where fluid particles move from one cell to another within one time step. If it is higher than one, fluid particles move through two or more cells at each time step and this can affect convergence negatively. For Co $=0.2, \Delta x=10^{-5} \mathrm{~m}, \sigma_{w o}=0.0003 \mathrm{~N} / \mathrm{m}, V_{o}=0.00009 \mathrm{~m} / \mathrm{s}, \rho_{o}=900 \mathrm{~kg} / \mathrm{m}^{3}$ and $\mu_{o}=10 \mathrm{cP}=0.01 \mathrm{~Pa} \cdot \mathrm{s}$, the values for the time step obtained by Eqs. (3.13), (3.14) and (3.15) 
are $\Delta t_{C F L}=0.002 \mathrm{~s}, \Delta t_{B}=2.185 \cdot 10^{-5} \mathrm{~s}$ and $\Delta t_{V}=3.33 \cdot 10^{-4} \mathrm{~s}$. In simulations performed in this study, it has been used $N=6900$ time steps of $\Delta t=0.002 \mathrm{~s}$, therefore the end of the simulation is at $t_{\text {final }}=13.8 \mathrm{~s}$.

The nine cases analysed with the values of the dimensionless parameters and the algorithms used are detailed in Table 2 . The Reynolds number is $R e=8.1 \cdot 10^{-4}$ for all the simulated cases.

Table 2. Parameters used in nine simulated cases

\begin{tabular}{|c|c|c|c|c|c|}
\hline Case & Algorithm & $\begin{array}{c}\text { Ca } \\
\text { Eq. (3.7) }\end{array}$ & $\begin{array}{c}\mu_{r} \\
\text { Eq. (3.8) }\end{array}$ & $\begin{array}{c}q_{R} \\
\text { Eq. (3.10) }\end{array}$ & Geometry \\
\hline \hline 1 & SIMPLE & 0.003 & 10 & 0.2158 & Fig. 1a \\
\hline 2 & SIMPLE & 0.00474 & 10 & 0.2158 & Fig. 1a \\
\hline 3 & SIMPLE & 0.01 & 10 & 0.2158 & Fig. 1a \\
\hline 4 & SIMPLE & 0.003 & 10 & 0.168 & Fig. 1a \\
\hline 5 & SIMPLE & 0.003 & 10 & 0.3 & Fig. 1a \\
\hline 6 & SIMPLE & 0.003 & 1 & 0.2158 & Fig. 1a \\
\hline 7 & SIMPLEC & 0.003 & 10 & 0.2158 & Fig. 1a \\
\hline 8 & PISO & 0.003 & 10 & 0.2158 & Fig. 1a \\
\hline 9 & SIMPLE & 0.003 & 10 & 0.2158 & Fig. 1b \\
\hline
\end{tabular}

For a simulation of $13.8 \mathrm{~s}$ (6900 time steps), each case takes approximately 56 minutes using a computer ASUS Intel ${ }^{\circledR}$ Core $^{\mathrm{TM}} \mathrm{i} 3-4005 \mathrm{U}$ CPU@1.7 GHz and $4 \mathrm{~GB}$ of RAM.

The convergence criteria is chosen to be $10^{-3}$ for the residual monitors. For this value, the convergence happens after 9 iterations on average.

\section{Results and discussions}

The results for all nine cases in Table 2 are explained in the following sections. For Subsections 5.1, 5.2 and 5.3, the physical domain used is defined by Fig. 1a and for Subsections 5.4, the one defined by Fig. 1b is used.

\subsection{Microdroplet formation process}

Figure 5 shows the time evolution of the droplet formation in the Y-junction for case 1. Initially, all the geometry is filled with water (white colour). From this moment, oil represented by black colour is injected into channel number 1 (according to notations in Fig. 1) with a constant velocity of $0.00009 \mathrm{~m} / \mathrm{s}$. In the meantime, water is continuously injected into channel 2 (according to notations in Fig. 1) with a constant velocity of $0.000417 \mathrm{~m} / \mathrm{s}$. The formation of droplets can be described as follows. The two immiscible fluids form an interface at the junction (Fig. 5a). The droplet begins to grow due to action of shear stress between the carrier and dispersed phases. Thus the neck width of the droplet becomes smaller until the droplet completely separates from the dispersed phase (Fig. 5b and Fig. 5c) forming two interfaces. At the broken time and due to capillary effects, these two interfaces displace in opposite directions at high velocities. One of the interface, which is always moving forward, forms the downstream meniscus of the droplet recently created. The other interface is moving backward (Fig. 5c) and because the fluid injection is constant, this negative velocity becomes zero and then positive. This process repeats itself periodically and a pattern of a droplet is formed in the main channel (Fig. 5d). The droplets areas equally spaced, have the same length and diameter larger than the main channel. These characteristics depend on the interfacial tension, viscosity ratio and flow rate ratio between the two fluids, besides of the geometry used. In this frame, it is also possible to observe the zero contact angle between the system oil/water/wall. 
(a)

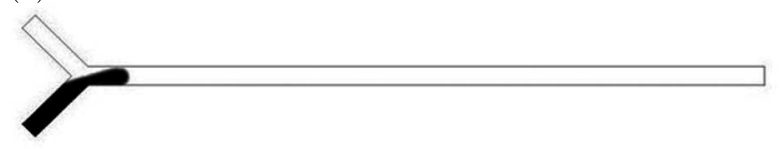

(c)

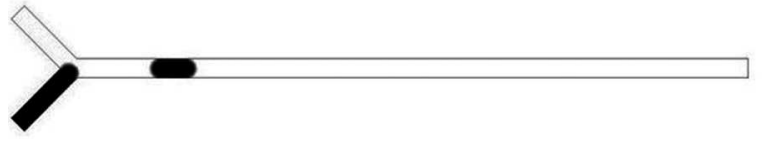

(b)

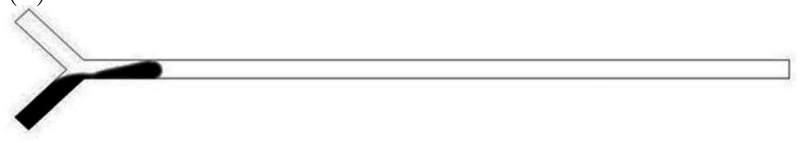

(d)

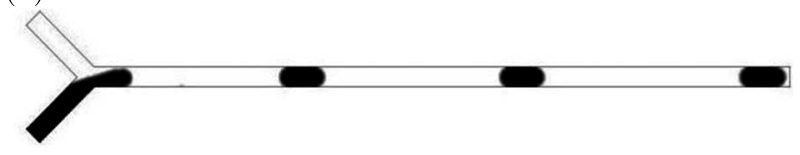

Fig. 5. Time evolution of droplet formation at $\mathrm{Ca}=3 \cdot 10^{-3}$ and $q_{R}=0.216$ at different time instants: (a) $6 \mathrm{~s}$, (b) $6.5 \mathrm{~s},(\mathrm{c}) 7 \mathrm{~s}$, and (d) $12.8 \mathrm{~s}$

The coherence of the previous results can also be explained by variation of pressure through the geometry. The contour of pressure is shown in Fig. 6 for four different time instants: $6 \mathrm{~s}$, $6.5 \mathrm{~s}, 7 \mathrm{~s}$ and $12.8 \mathrm{~s}$ which are also the same times used in Fig. 5.

(a) Pressure $[\mathrm{Pa}]$

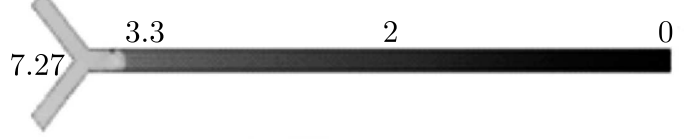

(c)

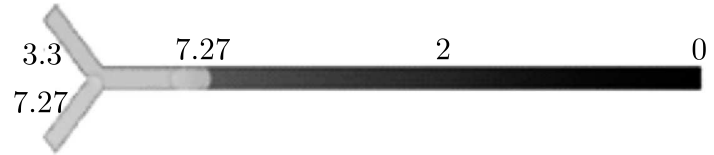

(b)

7.27

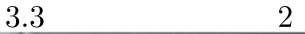

(d)

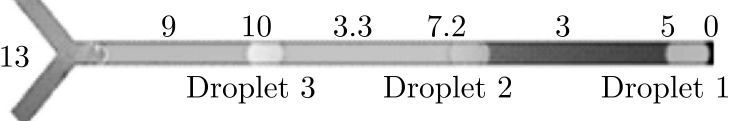

Fig. 6. Contour of pressure (in Pa) for the Y-junction at $\mathrm{Ca}=3 \cdot 10^{-3}$ and $q_{R}=0.216$ at different instants: (a) $6 \mathrm{~s}$, (b) $6.5 \mathrm{~s}$, (c) $7 \mathrm{~s}$ and (d) $12.8 \mathrm{~s}$

Figure 6a represents the contour of pressure at $6 \mathrm{~s}$ after the injection of the fluids, which is when the interface arrives to the junction. Because oil (channel 1) is ten times more viscous than water (channel 2), the pressure necessary to facilitate its movement through the channel is increasing, the pressure in the outlet is almost equal to zero, as set in the boundary conditions. When the dispersed fluid (oil) reaches the junction, the pressure drives a part of that fluid into the main channel and blocks the rest of the dispersed fluid from channel 1, as shown in Fig. 6b. Thus, as the carrier fluid (water) is moving through the secondary channel, a complete droplet is being formed. In Fig. 6c, the upstream pressure decreases once the droplet separates from the junction. This instantaneous drop in pressure happens because the blockage of the dispersed fluid stops and the second droplet starts forming. This formation process repeats periodically as both fluids are continuously injected. Figure 6d represents the contour of pressure when the first droplet reaches the outlet causing a small increase in the outlet pressure.

For this case, the contour of pressure is illustrated by a diagram in Fig. 7. The peaks in this figure represent the positions of droplets in the current instant $(t=13.8 \mathrm{~s})$ when they move along the main channel whose length is $0.0036 \mathrm{~m}(36 h$, with $h=0.1 \mathrm{~mm})$. One droplet is situated in a position $x=0.0015 \mathrm{~m}$ from the junction, almost half length of the channel, and another is at $x=0.0027 \mathrm{~m}$, close to the exit plane. The first droplet reaches the outlet, its pressure is equal to zero. In Fig. 7, it can be seen that the pressure in the droplet is as high as the droplet is close to the junction. This is due to the blockage exerted by the pressure, which has been explained previously. 


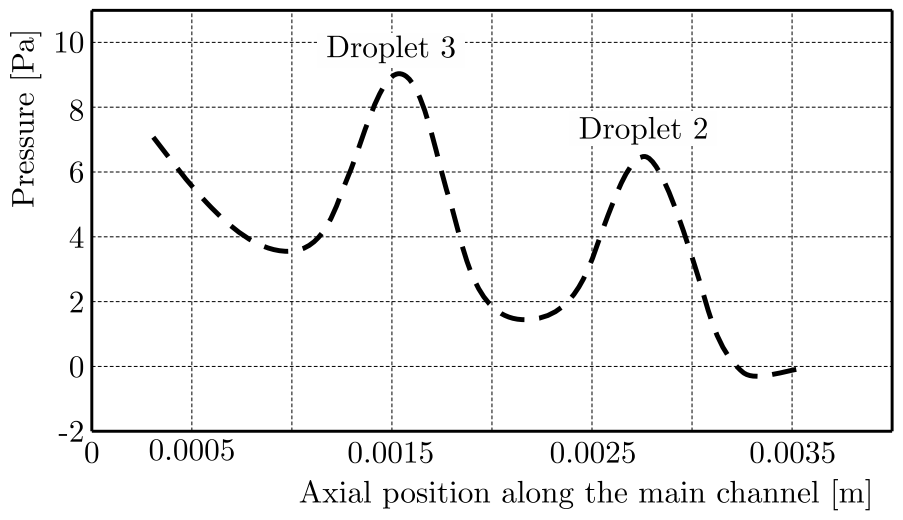

Fig. 7. Evolution of pressure in the main channel at the time instant $13.8 \mathrm{~s}$

\subsection{Dimensionless numbers effect}

\subsubsection{Capillary number}

The way in which droplets are formed is highly influenced by the capillary number. Simulations have been performed for three different values of the capillary number: cases 1, 2 and 3 (refer to Table 1).

For case 1 , with $\mathrm{Ca}=0.003$ the droplets or droplets generation occurs at the two-phase intersection and they have an elongated shape in the continuous phase microchannel. This regime is called "Squeezing regime" Tarchichi et al. (2013) (Fig. 8a). When Ca is increased, the droplets generated do not occupy the entire width of the continuous phase microchannel at the junction and they are smaller than the width of the continuous phase channel (Fig. 8b). This regime of droplet formation is known as "Dripping regime" (Tarchichi et al., 2013). In case 3, where $\mathrm{Ca}$ is increased again, the dispersed phase forms a long neck in the main channel (Fig. 8c). In this regime named "Jetting regime" (Tarchichi et al., 2013), the droplet or droplet formation occurs downstream at a some distance from the junction.

(a)

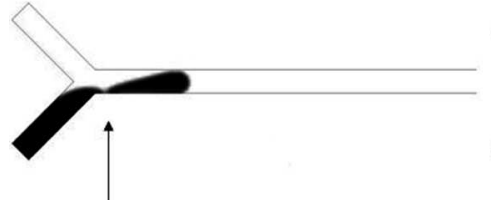

(b)

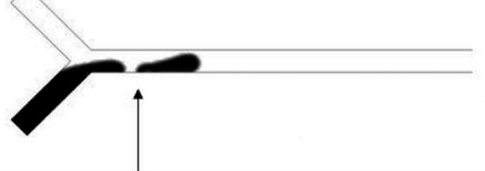

(c)

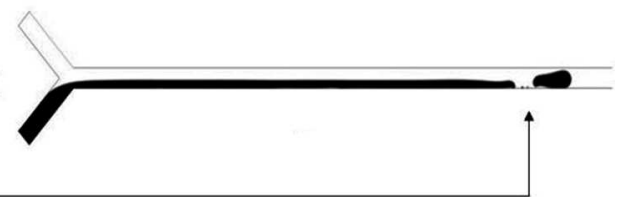

Position of the break up

Fig. 8. Position of droplet breakup for three regimes: (a) squeezing at $\mathrm{Ca}=0.003$, (b) dripping at $\mathrm{Ca}=0.00474$ and $(\mathrm{c})$ jetting at $\mathrm{Ca}=0.01$

\subsubsection{Flow rate ratio}

Here we investigate the influence of the flow rate ratio $\left(q_{R}=q_{o} / q_{w}\right)$ when the capillary number and the viscosity ratio are constant. Three different flow rate ratios are used: $q_{R}=0.168$, $q_{R}=0.2158$ and $q_{R}=0.3$ corresponding respectively to cases 1,4 and 5 , respectively. Figure 9 shows length of the droplet generated for each flow rate ratio. When $q$ is small (case 4), the droplets are pinched off at the junction. The length of the droplets in this case is $l=0.23 \mathrm{~mm}$. However when $q=0.2158$, the detachment point moves from the corner downstream (Fig. 9b). In this case, the droplets have a size equal to $0.25 \mathrm{~mm}$. When $q=0.3$, the length of the droplet is $l=0.26 \mathrm{~mm}$. 
(a)

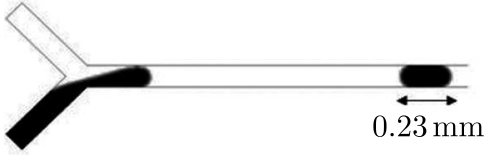

(b)

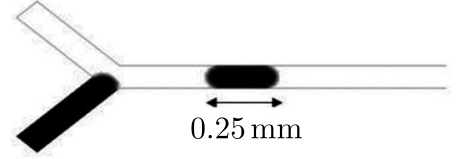

(c)

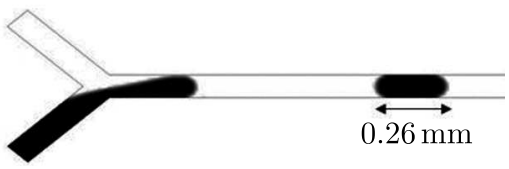

Fig. 9. Droplet length for the three flow rate ratios: (a) $q_{R}=0.17$, (b) $q_{R}=0.2158$ and (c) $q_{R}=0.3$

The results obtained confirm Eq. (3.11) which relates the size of the droplet with the flow rate ratio.

\subsubsection{Viscosity ratio}

Here we investigate the role of the viscosity ratio $\left(\mu_{R}=\mu_{o} / \mu_{w}\right)$ defined by Eq. (3.8) in the droplet formation process. The flow rate ratio and the capillary number are kept constant. Figure 10 shows the droplet formation for $\mu_{R}=10$ and $\mu_{R}=1$, respectively, at the same instant $t=12.8 \mathrm{~s}$. With a small viscosity ratio, the droplet formation occurs in the junction, which does not happen with a viscosity ratio equal to 10. Also, it can be seen in Fig. 10 that the decreasing viscosity ratio leads to smaller droplets with higher frequency. At the same instant, three droplets are formed for $\mu_{R}=10$, where we have four droplets for $\mu_{R}=1$. In fact, the breakup of the droplet is controlled by competition between viscous shear force and capillary force; which means when $\mathrm{Ca}$ is constant, the viscosity becomes a key factor in the droplet formation. That is why smaller droplets are obtained when $\mu_{R}=1$.

(a)

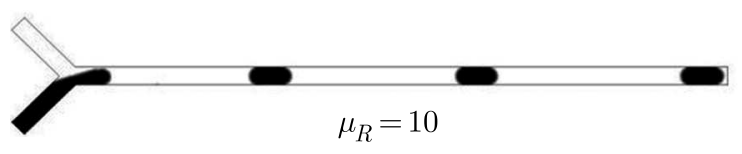

(b)

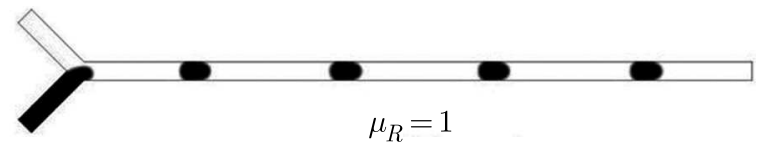

Fig. 10. Water volume fraction for two different viscosity ratios: (a) $\mu_{R}=10$ and (b) $\mu_{R}=1$

\subsection{Influence of discretization algorithms}

In this part, we investigate the effect of pressure-velocity schemes. Periodic droplets with a similar shape are obtained for all three algorithms (Fig. 11a, 11b and 11c). In this figure, it can be seen that the position of the droplet is little different from an algorithm to another. In fact,

(a)

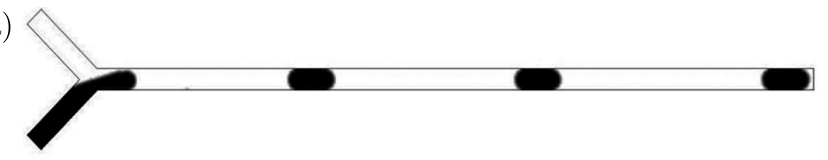

(b)

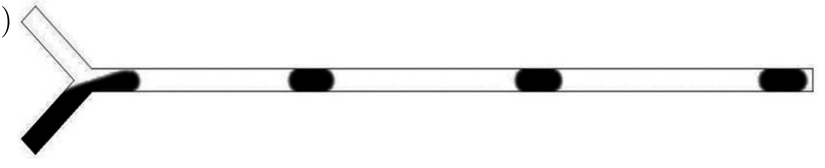

(c)

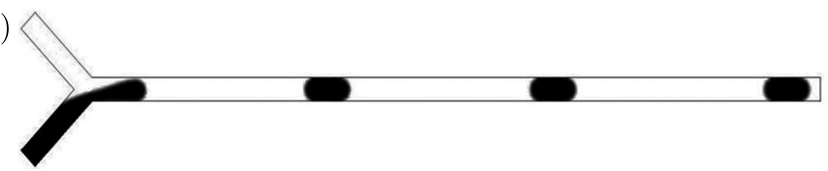

(d)

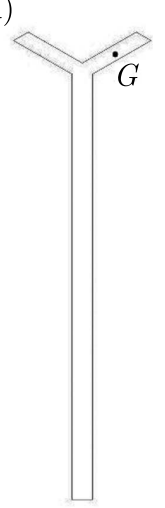

Fig. 11. Oil volume fraction for three algorithms: (a) SIMPLE, (b) SIMPLEC, and (c) PISO at the time instant $12.8 \mathrm{~s}$; (d) position of the center of gravity of the first inlet 
the difference between these algorithms lies in the pressure correction and the convergence speed, as has been mentioned in Section "Discretization algorithms". To have a better understanding of their effects, the time evolution of pressure is compared in each case. The pressure is measured at the same location (point $G$ ) which is the centre of gravity of channel 2 (Fig. 11d). This position is chosen because of the results in the contour of pressure shown in Fig. 6 and already commented. Indeed, the pressure in channel 2 varies periodically during the formation of a droplet. Thus, it would be easier to compare the effect of these algorithms in that position.

Figure 12 represents the time evolution of the pressure at the point $G$ for the three algorithms. In this figure, at the beginning, SIMPLE and SIMPLEC are more stable than PISO. However, from the third droplet ( $t=9.5 \mathrm{~s} ; N=4750$ time steps), PISO becomes more stable and accurate compared to the other algorithms, the oscillations amplitude in PISO is smaller than in the others. In fact, PISO is more recommended for simulation with large time steps, as has been mentioned in Section "Discretization algorithms". The results for SIMPLE and SIMPLEC are quite similar during the simulation. In fact, differences between the methods arise more from the single-phase flow. Also, the number of iterations for each time step is quite smaller in PISO than in the other algorithms. This leads to a faster simulation. All the results confirm that the algorithm PISO is better for a two-phase flow like that considered in our case.

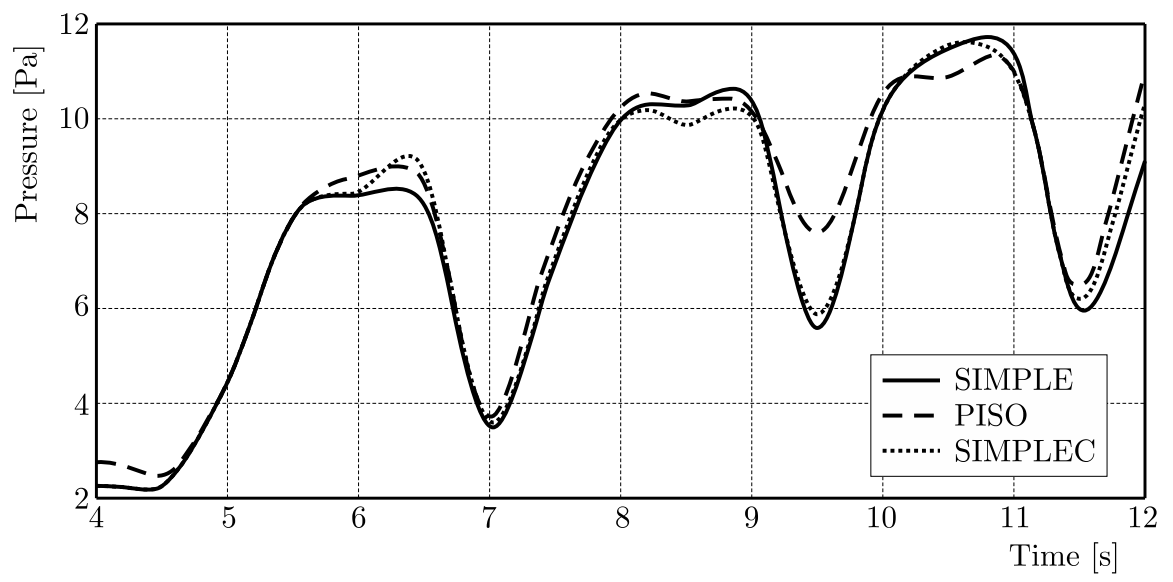

Fig. 12. Time evolution of pressure at the point $G$ (Fig. 11d) for the three algorithms SIMPLE, SIMPLEC and PISO

\subsection{Microdroplet breakup process}

In this part, the geometry described in Fig. 1a is modified by placing two orthogonal channels at the outlet, thereby forming another Y-junction but with length $10 h$ each. A theoretical mathematical point with zero radius of curvature represents the intersection between the two walls of the channels. The objective is to capture the breakup process of droplets formed in the first stage. Figure 13 represents the time evolution of the droplet breakup. When the droplet arrives at the junction, it is deformed symmetrically by the driving fluid (water in this case) in the center of the junction (Fig. 13a). Thus, the actions of the shear force and pressure drive the droplet to each outlet, thereby forming two half-droplets connected by the neck (Fig. 13b). From this moment, the neck becomes smaller until the breakup occurs (Fig. 13c). This process repeats itself when the droplet reaches the junction (Fig. 13d).

Figure 14 shows details of the breakup process with the velocity vectors in the junction. The uniform repartition of the velocity vectors in the two exit branches leads the droplet to form two identical liquid fingers in the upper and lower branch (Fig. 14a and Fig. 14b). When the droplet completely penetrates the branches, the droplet creates restriction to the fluid flow, being necessary a higher pressure to sustain the motion. Thus, a neck is formed at the junction, 

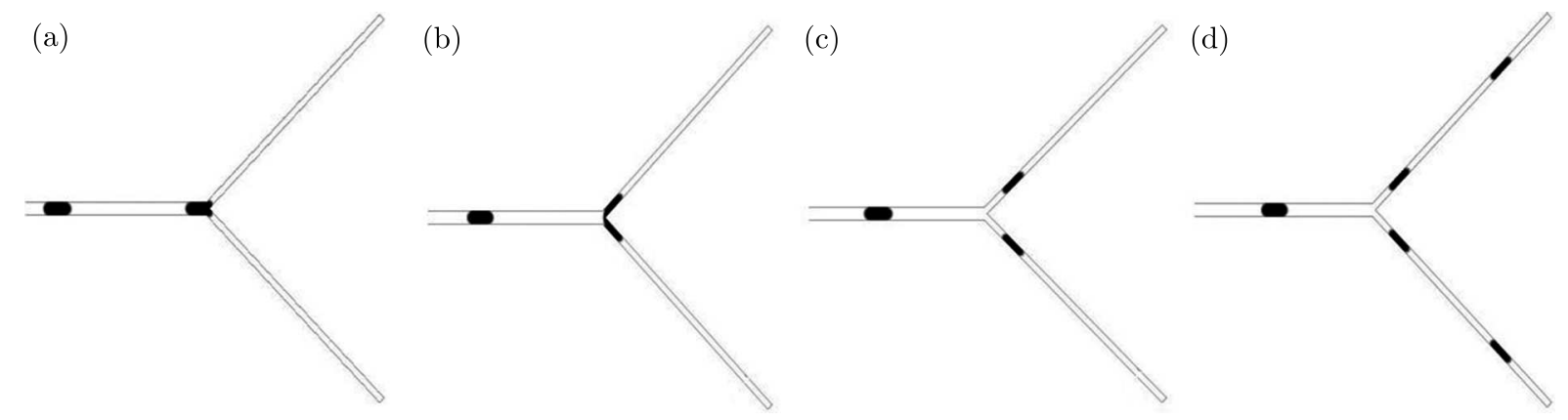

Fig. 13. Droplet breakup in the Y-exit
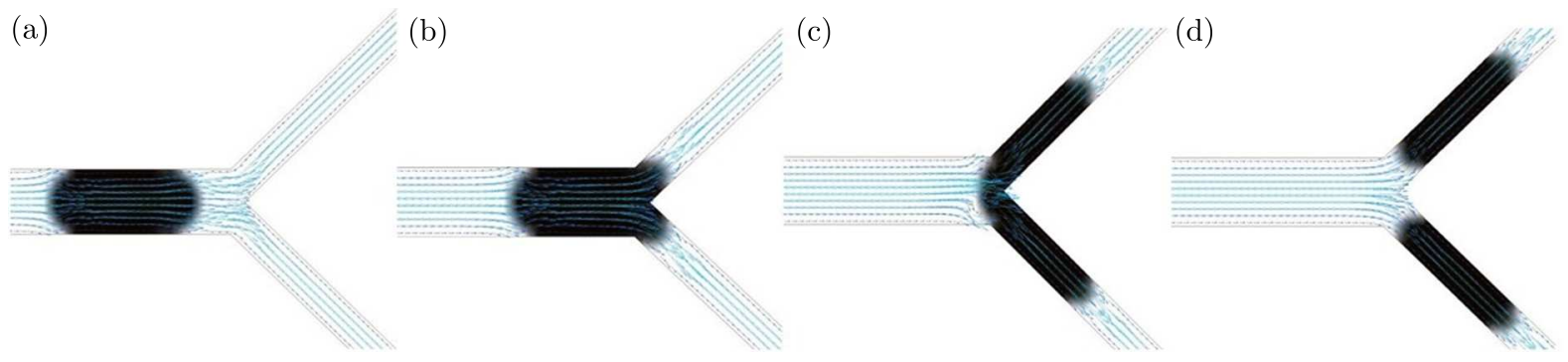

Fig. 14. Velocity fields during the droplet breakup

as it can be seen in Fig. 14c. As the droplet moves through the exit channels, the neck becames gradually smaller until the droplet breaks into two daughter ones. Once the breakup occurs, the restriction stops, thus the flow restarts normally and the two half-droplets are driven to in the outlets (Fig. 14d).

\section{Conclusions}

In the study, we investigate the formation process of droplets using geometry called Y-junction. The capillary number, the flow rate ratio and the viscosity ratio are found to be important in droplet formation, and these parameters together control the complex droplet generation process. Our results are in phase with what can be found in the literature. The analysis of pressure field shows that the pressure plays a major role in droplet formations.

The predicted length of droplets, as described by Tarchichi et al. (2013) and Garstecki et al. (2006) for the squeezing regime, is confirmed through simulations performed in this work, since the analytical and numerical values are almost the same.

The results of the simulations reveal that the algorithm PISO is the most suitable for a two phase flow problem, at least in the range of parameters considered in this work. SIMPLE and SIMPLEC do not show any particular difference in the results, due to the regime of the flows. However, the results for SIMPLE and SIMPLEC are also satisfactory.

The process of breaking the bubbles using a second Y-junction in the end shows a symmetric distribution of the droplets in the two outlet channels. The droplets divide into parts of equal size due to the same conditions in the two outlets $(0 \mathrm{~Pa})$. The dimensionless numbers also can affect the breakup process. In fact, a high value of the capillary number can lead to an asymmetric distribution.

\section{Acknowledgement}

The authors are thankful to international research program Capes/Brafitec (Coordenação de Aperfeiçoamento de Pessoal de Nível Superior/Brasil France Ingénieur Technologie) for providing the scholarship to carry out this study. 


\section{References}

1. Bethke C.M., 2008, Geochemical and Biogeochemical Reaction Modeling, Second Edition, Cambridge University Press, p. 297

2. Brackbill J.U., Kothe D.B., Zemach C., 1992, A continuum method for modeling surface tension, Journal of Computational Physics, 100, 335-354

3. Coelho J.K., Pena M.D., Romero O.J., 2016, Pore-scale modeling of oil mobilization trapped in a square cavity, IEEE America Latina, 14, 4, 1800-1807, DOI: 10.1109/TLA.2016.7483518

4. Cong Z., Zhu C., Fu T., MA Y., 2014, Bubble breakup and distribution in asymmetric Y-bifurcating microchannel, CIESC Journal, 65, 1, 93-99

5. Dolomite, 2015, Droplet microfluidics brochure for biology, food and cosmetics, drug discovery, chemistry, http://www.dolomite-microfluidics.com/en/applications

6. Ferziger J.H., Peric M., 2002, Computational Methods for Fluid Dynamics, Springer, Berlin

7. FLUENT, 2012, Ansys Inc. Fluent - Theory Guide Documentation

8. Fu T., Ma Y., Funfschilling D., Li H.Z., 2011, Dynamics of bubble breakup in a microfluidic T-junction divergence, Chemical Engineering Science, 66, 4184-4195

9. Fu T., MA Y., Li H.Z., 2014, Hydrodynamic feedback on bubble breakup at a T-junction within an asymmetric loop, AIChE Journal, 60, 5, 1920-1929

10. Galusinski C., Vigneaux P., 2008, On stability condition for bifluid flows with surface tension: Application to microfluidics, Journal of Computational Physics, 227, 6140-6164

11. Garstecki P., Fuerstman M.J., Stone H.A., Whitesides G.M., 2006, Formation of droplets and bubbles in a microfluidic T-junctionscaling and mechanism of break-up, Lab on a Chip, 6, 437-446

12. Hirt C.W., Nichols B.D., 1981, Volume of fluid (VOF) method for the dynamics of free boundaries, Journal of Computational Physics, 39, 201-225

13. Jamaloei B.Y., Kharrat R., Asghari K., 2011, The influence of pore wettability on the microstructure of residual oil in surfactant-enhanced water flooding in heavy oil reservoirs: Implications for pore-scale flow characterization, Journal of Petroleum Science and Engineering, 7, 1, 121-134

14. Lih F.L., Miao J.M., 2015, Effect of junction configurations on microdroplet formation in a T-Junction microchannel, Journal of Applied Mechanics and Technical Physics, 56, 2, 220-230

15. LiU Z.M., LiU L.K., FEnG S., 2015, Effects of geometric configuration on droplet generation in Y-junctions and anti-Y-junctions microchannels, Acta Mechanica Sinica, 31, 5, 741-749

16. PatankAR S.V., 1980, Numerical Heat Transfer and Fluid Flow (Series in computational methods in mechanics and thermal sciences), Hemisphere Publishing Corporation, Washington-New YorkLondon

17. Qian D., LAwal A., 2006, Numerical study on gas and liquid slugs for Taylor flow in a T-junction microchannel, Chemical Engineering Science, 61, 7609-7625, DOI: 10.1016/j.ces.2006.08.073

18. Santos K.B., Romero O.J., Meneguelo A.P., Ribeiro D.C., 2016, A numerical investigation of immiscible water-oil displacement in simplified porous media, IEEE America Latina, 14, 5, 2175-2183, DOI: 10.1109/TLA.2016.7530411

19. Tarchichi N., Chollet F., Manceau J., 2013, New regime of droplet generation in a T-Shape microfluidic junction, Journal of Microfluidics and Nanofluidics, 14, 1, 45-51

20. Tice J.D., Lyon A.D., Ismagilov R.F., 2004, Effects of viscosity on droplet formation and mixing in microfluidic channels, Analytica Chimica Acta, 507, 1, 73-77

21. Versteeg, H.K., Malalasekera W., 1998, An Introduction to Computational Fluid Dynamics, Longman, London

22. Waclawczyk T., Koronowicz, T., 2008, Comparison of CICSAM and HRIC high-resolution schemes for interface capturing, Journal of Theoretical and Applied Mechanics, 46, 2, 325-345 